\title{
An evaluation of the clinical diagnostic value of contrast-enhanced ultrasound combined with contrast-enhanced computed tomography in space-occupying lesions of the kidney [Corrigendum]
}

Tian W, Lu J, Jiao D, et al. Onco Targets Ther. 2017; 10:3493-3499.

On page 1, the author list and affiliation details were listed as:

Wen Tian ${ }^{1}$

Jianbo $\mathrm{Lu}^{2}$

Dan $\mathrm{Jiao}^{3}$

Zhibin Cong ${ }^{2}$

${ }^{1}$ Department of Blood Transfusion, The Second Hospital of Jilin University, ${ }^{2}$ Department of Ultrasound, Affiliated Hospital of Changchun University of Traditional Chinese Medicine, ${ }^{3}$ Department of Ultrasound, China-Japan Union Hospital of Jilin University, Changchun, Jilin, People's Republic of China
The authors advise that the author list and affiliation details should have been listed as:

Jianbo $\mathrm{Lu}^{1}$

Dan $\mathrm{Jiao}^{2}$

Zhibin Cong ${ }^{1}$

Wen $\operatorname{Tian}^{3}$

${ }^{1}$ Department of Ultrasound, Affiliated hospital of Changchun University of Traditional Chinese Medicine, ${ }^{2}$ Department of Ultrasound, China-Japan Union Hospital of Jilin University, ${ }^{3}$ Department of Blood Transfusion, The Second Hospital of Jilin University, Changchun, Jilin, People's Republic of China 